\title{
Overview on performance predictive models - Application to Bridge Management Systems
}

\author{
Monica Santamaria, João Fernandes, José C. Matos \\ Institute for Sustainability and Innovation in Structural Engineering (ISISE), University of Minho, \\ Guimarães, Portugal
}

Contacting author: monica.santamaria11@gmail.com

\begin{abstract}
Bridge management systems (BMSs) have been developed to assist the bridge asset engineers to determine the optimal cost-effective maintenance, rehabilitation, and replacement (MR\&R) decisions for bridge networks. The accuracy of these decisions depends significantly on the performance predictive models used to forecast the future condition of infrastructures. The most common performance predictive models used in the BMSs are deterministic and stochastic models. Several limitations in these models have been mentioned by many authors, which leads to a concern about the reliability of these models to effectively define the maintenance strategies. This paper presents an overview of the main performance predictive models that have been applied for infrastructures and recommends the implementation of some of these models in the BMSs.
\end{abstract}

Keywords: deterioration models, physical models, regression models, Markov models, Artificial Intelligence models, Bayesian networks, Petri Nets

\section{Introduction}

The importance of civil infrastructures for the development and economic growth of every country is a broadly accepted matter. Ensuring a satisfactory quality of the infrastructure assets along the network involves large capital investments in order to execute maintenance, rehabilitation and replacement actions. For this reason, each country is putting efforts in developing cost-efficient strategies to guarantee an adequate performance of the infrastructures, through the development of Bridge management systems (BMSs). A BMS is defined by Gatulli and Chiaramonte [1] as any system or collective series of engineering and management functions comprising the necessary actions to manage a bridge network. The successful of a BMS relies in determining the optimal intervention activities that assure structural safety and serviceability of the infrastructure assets, while investing the lowest financial costs. In order to accomplish this goal, one of the fundamental modules of the BMS are the performance predictive models. Their purpose is to forecast the deterioration of the infrastructures along time. Hence, the subsequent modules regarding the time and the extent of the maintenance actions needed, depend entirely on the deterioration model established.

The International Association for Bridge Maintenance and Safety (IABMAS) committee compiled an overview report on the existing bridge management systems around the world. The third version of this report was based on questionnaires on 25 BMS from 18 countries [2]. Nineteen of the analysed systems could predict deterioration, among which twelve of these systems were using probabilistic methods. This paper presents an overview of the predictive models that are stated to be used in current BMSs, together with some other models that have been proposed in the literature for modelling bridge deterioration, which 
could represent a potential alternative for the prediction module of BMSs.

\section{Physical models}

The degradation of a structure is a response to the interaction of different physical processes within the system, subjected to certain environmental conditions [3]. Physical models of mechanical and chemical processes have been developed to represent the structure deterioration. For instance, the fib Bulletin 65 [4] present some broadly accepted models for concrete structures, describing damaging processes where external substances cause deterioration by direct action such as freeze/thaw attack, reactivity of aggregate and/or cement paste, acid action and leaching processes; or indirectly, allowing other reactions to cause deterioration such as carbonation-induced corrosion of reinforcing steel and chloride-induced corrosion of reinforcing steel.

According to IABMAS questionnaires [2], chloride, carbonation and corrosion deterioration models are implemented in the German Bridge Management System (GBMS). Likewise, as reported by Zambón et al [5], the Austrian BMS called LeCIE_tool currently being developed, is predicting the remaining service life of existing concrete bridges based on chloride- and carbonation-induced corrosion models, since they have been identified as main causes of infrastructure deterioration [5].

The carbonation progress formulation in the fib Bulletin 65 [4] is expressed as:

$x_{c}(t)=\sqrt{2 k_{e} k_{c} R_{N A C, 0}{ }^{-1} C_{s}} \sqrt{t} W(t)$

Where

$x_{c}(t)$ is the carbonation depth at the time $t$ in $\mathrm{mm}$;

$t$ is the time in years;

$k_{e}$ is the environmental function [-];

$k_{c}$ is the execution transfer parameter [-];

$C_{S}$ is the $\mathrm{CO}_{2}$ - concentration in the air in $\mathrm{kg} / \mathrm{m}^{3}$;

$W(t)$ weather function [-];

$R_{N A C, 0}{ }^{-1}$ is the inverse effective carbonation resistance of concrete in $\left(\mathrm{mm}^{2} /\right.$ years $) /\left(\mathrm{kg} / \mathrm{m}^{3}\right)$;
In the same line, the ingress of chlorides formulation according to fib Bulletin 65 [4] is described by:

$C(x, t)=C_{0}+\left(C_{S, \Delta x}-C_{0}\right)\left[1-\operatorname{erf} \frac{x-\Delta x}{2 \sqrt{D_{a p p, C}}}\right](2)$

Where

$C(x, t)$ is the chloride content of concrete in \% by mass of cement;

$x$ is the depth in $\mathrm{m}$;

$t$ is the concrete age in s;

$C_{0}$ is the initial chloride content in $\%$ by mass of cement;

$C_{S, \Delta x}$ is the chloride content at a depth of $\Delta x$ in \% by mass of cement;

$\Delta x$ is the depth of the convection zone in $\mathrm{m}$;

$D_{a p p, C}$ is the apparent chloride diffusion coefficient in concrete in $\mathrm{m}^{2} / \mathrm{s}$;

The advantage of these models is that they consider particular phenomena causing deterioration [5]. However, the disadvantage relies in the fact that is necessary to determine the exact parameters of each bridge, because there is a high sensitivity in the results (predicted service life) when slightly modifying the input of crucial parameters [5].

\section{Regression models}

Degradation models should be established based on data from field observations and the knowledge of the mechanical laws that drive the system performance [3]. However, the mathematical formulation to describe the degradation processes usually comprise high complexity, so degradation models frequently rely only on field data [3].

Regression models can be considered as one of the simplest infrastructure deterioration models employing measurements data. These models establish an empirical relationship between two or more variables; e.g. the data of a bridge's condition and the factors affecting their deterioration [6].

The Korean Road Maintenance Business System (KRMBS) reported to the IABMAS questionnaires [2] the application of deterioration models based on regression of historical condition state data. The 
output of these models is expressed by deterministic values that represent the average predicted condition [6]. Several research studies have applied these deterministic methods such as straight-line extrapolation, linear, non-linear and stepwise regressions, and curve-fitting techniques to model infrastructure deterioration $[6,7]$. However, these techniques disregard the uncertainty involved on the variables, they do not account for the current condition and the condition history of a facility in predicting the future one, and they ignored the interaction between degradation of different infrastructure components [8], they merely fit the best line/curve to the data. In consequence, for predicting a short period of time they are acceptable, but when these functions are projected beyond the bounds of the data for long time periods (which is required in the case of systems with expected long lifetimes such as civil infrastructures), they can contain high levels of inaccuracy [7].

\section{Stochastic models}

The deterioration of bridges is a complex process resulting from the interaction of several damage mechanisms, progressing at different rates and in some cases unobservable. In consequence, the parameters which define the deterioration processes are always affected by uncertainties, hence prediction models should be formulated in probabilistic terms [9]. For this reason, stochastic models have been widely used for modelling bridge deterioration, because they treat the degradation as one or more random variables that capture the uncertainty and randomness of the process [8].

The most commonly used stochastic models for predicting infrastructure deterioration are Markov models. In the survey description of the IABMAS report, eight of the BMSs stated to apply Markovian deterioration models. A significant reason for selecting these models is that in BMSs discrete ratings are used instead of continuous indices, because they must manage large amounts of assets that otherwise will represent a high computational cost. Consequently, discrete state degradation models such as Markov models have been preferred due to their suitability to use bridge condition data and their computational efficiency to manipulate networks [10].

\subsection{Markov models}

Markov models are stochastic processes that represent observations on physical systems satisfying the condition that the probability that the system will be in a given state at a time $t_{2}$, may be deduced from a knowledge of its state at any earlier time $t_{1}$, and does not depend on the history of the system before time $t_{1}$ (i.e. memoryless property) [11]. A Markov chain is a special case of the Markov process, where the number of possible states is either finite or countably infinite (discrete state space), and whose development holds the Markovian property [11]. For a system observed at a discrete set of times, the conditional probability of moving into state $j$ at time $n+1$ given that at time $n$ the object is in state $i$ is expressed by [12]:

$P_{i j}=\operatorname{Pr}\left\{X_{n+1}=j \mid X_{0}=i_{0}, \ldots, X_{n-1}=\right.$

$\left.i_{n-1}, X_{n}=1\right\}=\operatorname{Pr}\left\{X_{n+1}=j \mid X_{n}=1\right\}$

Markov chains are used in the BMSs as performance prediction models by defining discrete condition states corresponding to the bridge elements condition ratings, and accumulating the probability of transition from one condition state to another over multiple discrete time intervals [10]. Transition probabilities are represented by a transition probability matrix (TPM) of order ( $n \times n)$, where $n$ is the number of possible condition states:

$P=\left[\begin{array}{cccc}P_{11} & P_{12} & \cdots & P_{1 N} \\ P_{21} & P_{22} & \cdots & P_{2 N} \\ \vdots & \vdots & \ddots & \vdots \\ P_{N 1} & P_{N 2} & \cdots & P_{N N}\end{array}\right]$

Each element in the matrix represents the probability that the bridge element condition will change from state $i$ to state $j$ during the transition period [10].

The derivation of the transition probabilities is of utmost importance for the Markov Chain models. There are different methods to estimate the TPM, among which two of the most common are the percentage prediction method and the regressionbased optimization method.

In the percentage prediction method, the probability $p_{i j}$ is estimated by [13]: 
$p_{i j}=\frac{n_{i j}}{n_{i}}$

Where:

$n_{i j}$ is the number of bridges that moved from state $i$ to state $j$ during one cycle;

$n_{i}$ is the total number of bridges in state $i$ before the transition

On the other hand, in the regression based optimization method the transition probabilities can be estimated by solving the nonlinear optimization problem that minimises the sum of absolute distance between the regression curve that best fits the condition data and the conditions predicted using the Markov chain model [10]. The objective function and the constraints can be formulated as:

$\min \sum_{i=1}^{N}|Y(t)-E(t, P)|$

Where

$\mathrm{N}=$ total number of transition periods;

$Y(t)=$ condition at transition period number $t$ based on the regression curve;

$E(t)=$ expected condition at transition period number $t$ based on Markov chains;

$E(t)=P(t) S$

$\mathrm{S}=$ vector of condition states;

$P(t)=$ Transition probability matrix;

One of the main drawbacks in most of the BMS is the absence of historical condition data. For this reason, the advantage that Markov models provides is the possibility of building a forecasting model with a minimum of two visual inspections, given that the probability of the future condition depends only on the present condition.

Despite the widespread use of Markov models among the BMS, the following limitations in the application of these models compromise the accuracy of the predictive results:

- Markov chain theory requires the parameters to be stationary in time (or homogeneous), which implies that the TPM is fixed or constant throughout the predictive period [13], i.e. the rates of transition from one state to another remain constant throughout the time [14]. In this manner, the transition probabilities depend only on the current state and not on the ages of the bridges (age-independent assumption), thus a 5-year old bridge and a 80-year old one, both in the same condition state, have the same probability of changing to the next condition state [14]. Considering that aging is a property in which the probability of a structure to deteriorate to a lower state increases with time [15], several authors have proposed using a probability distribution with increasing failure rate for the uncertain time spent in each condition state (see Kleiner [16]).

- The memoryless property that implies the deterioration is independent of history may be unrealistic [17]. For example, early initiation of damage mechanisms could lead to accelerated deterioration in later stages [17]. Morcous [10] validated this assumption using a frequency test and inference test. The results showed that this assumption may not be acceptable for project level analysis where accurate prediction of element distresses is required. However, is acceptable for network level analysis with a $95 \%$ level of confidence [10].

- Transportation agencies inspect their bridges according to the severity of the conditions previously found, and the costs and benefits involved in performing the inspections [10]. Hence, the condition data is obtained with unequally spaced inspection periods, which is not adequate to develop the Markov chain models that assume discrete transition time intervals with constant inspection period [10]. According to Morcous [10] the variation in the inspection period may result in a $22 \%$ error in predicting the service life of a bridge deck system.

- The historical data in the BMS resulting from monitoring and visual inspections often contains incomplete data and measurement errors arising from defects on monitoring devices, from data processing and data interpretation [18], which cause bias in the prediction results. For instance, the subjectivity in the inspection process was documented in a research study conducted on 49 bridge inspectors providing condition ratings to the same bridges [19]. The results were normally distributed, and it was found that $68 \%$ of the ratings fell within an interval of \pm 1 rating point 
around the mean, and $95 \%$ fall within \pm 2 rating points around the mean.

- Markov models employ the available condition ratings data for modelling bridge deterioration. However, condition ratings are not accurate measures of the structural safety and serviceability; they are a qualitative performance indicator that allows to identify the most deteriorated bridges in a network, but they do not provide a quantitative evaluation of the bridge remaining capacity nor the improvement needed [20].

- Relying on visual inspection data to build the forecasting models undergoes a limitation in the prediction of the worst condition states, because there is generally an unbalanced distribution of the records. This data unbalance arises since the number of cases presenting severe damage are relatively low in comparison with the large number of cases reporting relatively good condition, which is expected considering that elements with poor structural condition will be repaired or replaced [21].

\subsection{Weibull survival functions}

Nowadays, the North American system AASHTOWare is considered one of the most complete and popular BMS in the world. For the element deterioration module, AASHTOWare recently developed an enhancement by combining Weibull survival functions to model the transition from condition state 1 to condition state 2 (i.e. the initiation of deterioration); and Markovian processes to model the rest of the transitions [22]. The implementation of Weibull survival functions seeks to consider the slower deterioration rates in the early stages. This is possible given that these models assume that the variable $T_{i}$ which represents the duration in number of years that an element stays in a particular condition rating, is a random variable modeled by a Weibull distribution [23]. Therefore, the probability that $T_{i}$ exceeds $t$ years, is the survival function of $T_{i}$ expressed by [23]:

$S_{i}(t)=e^{-\left(t / \eta_{i}\right)^{\beta_{i}}}, t>0, \beta_{i}>0, \eta_{i}>0, i=$

$7,6, \ldots, 1$

Where $\beta_{i}$ is the shape parameter of the Weibull function $\eta_{i}$ is the scale parameter of the Weibull function $i$ is the condition rating according to the predefined scale

The shape parameter determines if the hazard rate is decreasing $\left(\beta_{i}<1\right)$, constant $\left(\beta_{i}=1\right)$, or increasing $\left(\beta_{i}>1\right)$ [23]. Weibull distribution parameters can be obtained by fitting the duration data for each condition rating.

\section{Discussion on additional deterioration models}

The previous chapters presented a brief formulation of the deterioration models that have been employed by the BMSs according to the IABMAS questionnaires, and the available literature found regarding the late development of the transportation systems. Some of the advantages of applying each model were stated, as well as their limitations. A great amount of research has been conducted with the purpose of overcoming the existing restrictions of the models. In this subsection, some of those approaches will be briefly mentioned with the explanation of their potential capabilities to replace existing models in the BMSs.

\subsection{Reliability-based models}

In order to address the bridge performance from a quantitative evaluation instead of a condition state representing the damage, a significant amount of studies have used the reliability index as a measure of the performance of a bridge. Under no maintenance scenario, the time-dependent reliability index is described by [12] :

$\beta(t)=\left\{\begin{array}{cl}\beta_{0} & \text { for } 0 \leq t \leq t_{I}, \\ \beta_{0}-\alpha_{1}\left(t-t_{I}\right) & \text { for } t>t_{I}\end{array}\right.$

Where

$\beta(t)$ is the time-dependent reliability index

$\beta_{0}$ is the initial reliability index

$t_{I}$ is the time of initiation of deterioration

$\alpha_{1}$ is the deterioration rate of reliability index

Nonlinear reliability index models have been also proposed to capture the nonlinear effects of the 
deterioration processes [12]. Furthermore, timedependent reliability index models have been extended to account for the effect of different maintenance actions in the performance of deteriorating structures [12].

\subsection{Artificial Intelligence models}

Artificial intelligence (AI) models are techniques that enable the development of prediction models by learning from data and mimicking human intelligence [21]. Therefore, Al models take advantage of the computer capabilities that aim to automate intelligent behaviours [8].

Several research efforts have been made since the 1990s to implement Al models in bridge prediction modelling. Among the Al applications in this area, different types of Artificial Neural Networks (ANN) and case-based reasoning (CBR) are the most employed techniques. For a brief summary of the Al applications to bridge deterioration modelling refer to Zhe $\mathrm{Li}$ et al [21]. In general, research outcomes have demonstrated that Al models have a better performance than current bridge deterioration models in BMSs. For instance, when using an ensemble of neural networks approach (ENN) it was possible to identify damage with an accuracy of $86 \%$ [21]. However, as far as authors' knowledge goes, they have not yet been implemented in the existing BMS.

\subsection{Bayesian networks models}

A Bayesian network (BN) represents the joint probability of a set of random variables [24], which are linked under certain dependencies between them. Those relationships can be represented by a directed acyclic graph [25] where the variables are nodes, and the arrows directions designate the parents and the children. Figure 1 presents an example of a simple BN, where the child node is the bridge condition, which has a conditional dependence on two parent nodes, the environmental condition and the material type.

The joint probability of the variables in the network is calculated as the product of the conditional probability of each variable $X_{i}$ given its parents, which is expressed by [24]:

$p(X)=\prod_{i=1}^{n} p\left(X_{i} \mid\right.$ parents $\left.\left(X_{i}\right)\right)$

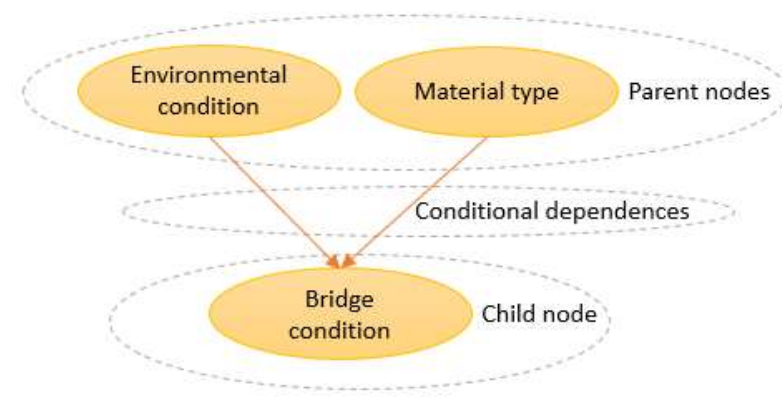

Figure 1. BN representation

Accordingly, the BN has two components [24]: the acyclic graph of the variables with their dependencies, and the conditional probability distribution of each variable represented by a set of conditional probability tables (CPTs).

There are some approaches in the literature for predicting the condition of bridge elements through BNs (see Wang et al [25]). The proposed model stated improved predictive results given the capacity of the method of considering multiple factors in the deterioration process. Furthermore, one of the principal advantages of BNs is its inference ability [25]; i.e. when a variable has a known state as a result of new observed evidence, an inference algorithm can update the probability distribution of the remaining variables, using Bayes' theorem [24]. This capability could be valuable in BMSs, where the databases are regularly updated with new inspection records.

\subsection{Petri-Nets models}

The Petri-Net (PN) model can be defined as a directed graph with two type of nodes: places and transitions, which are connected by arcs [26]. The third feature of the PN model are the tokens, which are located in the places so they define the current state of the system [26]. In this manner, a transition allows the token to move from one place to another, thus the dynamic behaviour of the system is modelled [26].

Applied to bridge deterioration modelling, places can represent a condition state of the rating system, tokens represent the bridge or the bridge element, while transitions represent the movement between condition states, therefore simulating deterioration trough a sequence of places and stochastic timed transitions [27] (see Figure 2). 


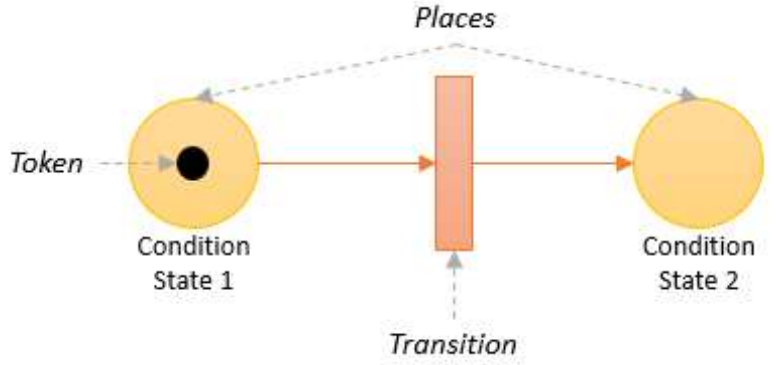

Figure 2. PN representation

In the last years, some studies have addressed the bridge deterioration modelling through PN models. These approaches found the advantage of modelling individual components in the structure, hence taking into consideration the interaction and dependency between multiple element deterioration processes, together with the maintenance process [26].

\section{Conclusions}

This paper provided a review of different deterioration modelling approaches that have been applied for predicting bridge performance over time. In the first part, the paper presented a brief formulation of the currently used models among the BMSs, namely physical models, regression models and Markov models, and presented some of the disadvantages of applying these models. In the second part, the paper summarized additional modelling techniques that have been developed as an attempt to overcome some of the limitations of the existing models. In a general sense, condition-based models have been always criticized due to their highly dependence on the quality of the condition ratings documented in the databases. However, reliability-based methodologies that could account for the reduction of the structural capacity of the bridge without considering the information from visual inspections, have not been attractive by the managers due to the fact of discarding condition states as a performance indicator in the BMSs. For this reason, the latest approaches have focused of employing the condition states but in a more efficient way than Markov models, therefore considering dependence relationships between the condition and identified deteriorating factors, considering interaction between degradation of different bridge components, implementing simple methods to update the models when new data is obtained, accounting for maintenance activities, among other features; leading all of them to a superiority in the prediction accuracy.

\section{Acknowledgements}

The authors would like to thank ISISE - Institute for Sustainability and Innovation in Structural Engineering (PEst-C/ECI/UI4029/2011 FCOM-010124-FEDER-022681) and FCT - Portuguese Scientific Foundation for the research grant PD/BD/128015/2016 under the PhD program "Innovation in Railway System and TechnologiesiRail"

\section{References}

[1] Gattulli V, Chiaramonte L. Condition assessment by visual inspection for a bridge management system. Computer-Aided Civil and Infrastructure Engineering. 2005;20(2):95-107.

[2] Mirzaei Z, Adey BT, Thompson P, Klatter L, editors. Overview of existing Bridge Management Systems-Report by the IABMAS Bridge Management Committee (2014)2014: International Association for Bridge Maintenance And Safety (IABMAS).

[3] Sánchez-Silva M, Klutke G-A. Reliability and life-cycle analysis of deteriorating systems: Springer; 2016.

[4] Code M. First complete draft, vol. 1. fib Bulletin. 2010(55).

[5] Zambon I, Vidocic A, Strauss A, Matos JC, Friedl N. Prediction of the remaining service life of existing concrete bridges in infrastructural networks based on carbonation and chloride ingress. 2018.

[6] Hatami A, Morcous G. Developing deterioration models for Nebraska bridges. 2011.

[7] Zayed TM, Chang L-M, Fricker JD. Statewide performance function for steel bridge protection systems. Journal of performance of constructed facilities. 2002;16(2):46-54. 
[8] Morcous G, Rivard H, Hanna A. Modeling bridge deterioration using case-based reasoning. Journal of Infrastructure Systems. 2002;8(3):86-95.

[9] Biondini F, Frangopol DM. Life-cycle performance of deteriorating structural systems under uncertainty. Journal of Structural Engineering. 2016;142(9):F4016001.

[10] Morcous G. Performance prediction of bridge deck systems using Markov chains. Journal of performance of Constructed Facilities. 2006;20(2):146-55.

[11] Parzen E. Stochastic processes: SIAM; 1999.

[12] Frangopol DM, Kallen MJ, Van Noortwijk JM. Probabilistic models for life-cycle performance of deteriorating structures: review and future directions. Progress in Structural Engineering and Materials. 2004;6(4):197-212.

[13] Collins L, editor An introduction to Markov chain analysis1975: Geo Abstracts Ltd.

[14] Lounis Z, Mirza M. Reliability-based service life prediction of deteriorating concrete structures. 2001.

[15] Kallen M, Van Noortwijk J, editors. A study towards the application of Markovian deterioration processes for bridge maintenance modelling in the Netherlands. Advances in Safety and Reliability: Proceedings of the European Safety and Reliability Conference (ESREL 2005), Poland; 2005.

[16] Kleiner Y. Scheduling inspection and renewal of large infrastructure assets. Journal of infrastructure systems. 2001;7(4):136-43.

[17] Madanat SM, Karlaftis MG, McCarthy PS. Probabilistic infrastructure deterioration models with panel data. Journal of infrastructure systems. 1997;3(1):4-9.

[18] Kobayashi K, Kaito K, Lethanh N. A statistical deterioration forecasting method using hidden Markov model for infrastructure management. Transportation Research Part B: Methodological. 2012;46(4):544-61.
[19] Phares BM, Rolander DD, Graybeal BA, Washer GA. Reliability of visual bridge inspection. Public Roads. 2001;64(5).

[20] Ng S, Moses F. Prediction of bridge service life using time-dependent reliability analysis. Bridge management. 2014;3:26-33.

[21] Li Z, Burgueño R. Using soft computing to analyze inspection results for bridge evaluation and management. Journal of Bridge Engineering. 2010;15(4):430-8.

[22] Johnson J. AASHTOWare BrM 5.2.3 Deterioration and LCCA. 2017 April 26, 2017.

[23] Agrawal AK, Kawaguchi A, Chen $Z$. Deterioration rates of typical bridge elements in New York. Journal of Bridge Engineering. 2010;15(4):419-29.

[24] Zhang H, Marsh DWR. Generic Bayesian network models for making maintenance decisions from available data and expert knowledge. Proceedings of the Institution of Mechanical Engineers, Part O: Journal of Risk and Reliability. 2018;232(5):505-23.

[25] Wang R, Ma L, Yan C, Mathew J, editors. Condition deterioration prediction of bridge elements using Dynamic Bayesian Networks (DBNs). Quality, Reliability, Risk, Maintenance, and Safety Engineering, International Conference; 2012: IEEE.

[26] Le B, Andrews J. Petri net modelling of bridge asset management using maintenancerelated state conditions. Structure and Infrastructure Engineering. 2016;12(6):73051.

[27] Yianni PC, Rama D, Neves LC, Andrews JD, Castlo D. A Petri-Net-based modelling approach to railway bridge asset management. Structure and Infrastructure Engineering. 2017;13(2):287-97. 